\title{
Prilocaine/lidocaine patch as a local premedication for skin biopsy in children
}

\author{
Flora B. de Waard-van der Spek, MD, ${ }^{a}$ Paul G. H. Mulder, PhD,${ }^{b}$ and \\ Arnold P. Oranje, MD, PhD ${ }^{\mathrm{a}}$ Rotterdam, The Netherlands
}

Background: Prilocaine-lidocaine emulsion (EMLA) has been used successfully as a local anesthetic in patients undergoing superficial procedures.

Objective: The purpose of this study was to investigate the analgesic effect of the patch as a local anesthetic for children undergoing a skin biopsy procedure. The effect on both the pain at the injection site and the pain of the biopsy was investigated.

Methods: The study was randomized, double blinded, and placebo controlled. Of the 63 children randomly recruited into the study, 60 children were evaluated; the remaining three were withdrawn from the study.

Results: The anesthetic patch was significantly more effective than the placebo patch in decreasing pain at the injection site. No significant difference in pain scores of the biopsy procedure was found between the study groups. No serious side effects were observed. Conclusion: The anesthetic patch reduced the pain experienced by children subjected to a skin biopsy procedure.

(J Am Acad Dermatol 1997;37:418-21.)

A skin biopsy procedure usually requires the infiltration of a local anesthetic, a process that itself is often painful and causes anxiety. Use of topical prilocaine-lidocaine cream (EMLA) as a local anesthetic has been successful in patients undergoing superficial procedures such as venipuncture, lumbar punctures, and curettage of mollusca contagiosa..$^{1-5}$ Use of this cream as a local anesthetic in patients undergoing a skin biopsy procedure has been examined. In a study of 12 adult volunteers, the maximal depth of anesthesia achieved with use of the cream was $5 \mathrm{~mm}$; this depth was reached 30 minutes after application of the cream for 90 minutes and 60 minutes after application for 120 minutes. ${ }^{6}$ In another study, 10 patients reported pain deep in the dermis during a skin biopsy procedure after application of the cream. ${ }^{7}$ In a study by Jones et al., ${ }^{8}$ a 60 -minute application of cream was not effective in elimi-

\footnotetext{
From the Department of Dermatology and Venereology, University Hospital Rotterdam, ${ }^{a}$ and the Department of Epidemiology \& Biostatistics, Erasmus University, Rotterdam. ${ }^{\text {b }}$

Reprint requests: F. B. de Waard-van der Spek, Department of Dermatology and Venereology, University Hospital Rotterdam, Dr. Molewaterplein 60,3015 GJ Rotterdam, The Netherlands.

Copyright () 1997 by the American Academy of Dermatology, Inc. 0190-9622/97/\$5.00+0 $\quad \mathbf{1 6 / 1 / 8 3 2 0 3}$
}

nating the pain caused by injection of a local anesthetic in 60 adult patients. To enhance the ease of application, a single unit dose package of lidocaine/prilocaine $5 \%$ emulsion has been developed, which might eliminate the possibility of incorrect application that leads to lack of analgesic effect. In a study of 108 adults undergoing either excisional biopsy procedures or curettage with electrosurgery, EMLA cream (Tegaderm) and patch formulations were shown to be equally effective"; this study also showed that the cream provided effective anesthesia in $87 \%$ of subjects after application for 110 to 180 minutes.

No reports exist of serious side effects after application of the anesthetic emulsion, although a temporary local redness or paleness of the skin may occur.

The purpose of this study was to investigate the analgesic effect of the patch as a local anesthetic for children undergoing a skin biopsy procedure. The effect on both the pain at the injection site and the pain of the biopsy was investigated. We also evaluated the differences in pain experienced by boys and girls.

\section{MATERIAL AND METHODS}

The protocol for the study was approved by the Medical Ethical Committee of the University Hospital 
Table I. Statistical analysis of the difference in VAS scores after the injection and the biopsy between the EMLA group and the placebo group

\begin{tabular}{|c|c|c|c|c|}
\hline & \multicolumn{2}{|c|}{ Injection } & \multicolumn{2}{|c|}{ Biopsy } \\
\hline & EMLA & Placebo & EMLA & Placebo \\
\hline Median & 0.80 & 2.80 & 0 & 0 \\
\hline IQR & $0.13-2.25$ & $1.23-6.65$ & $0-0.45$ & $0-2.03$ \\
\hline $\begin{array}{l}\text { Mann-Whitney } \\
\text { test } p \text { value }\end{array}$ & & & & \\
\hline
\end{tabular}

$I Q R$, Interquartile range.

Table II. Distribution of the verbal pain scores of the patients and the physician and statistical analysis of the difference in verbal pain scores after the injection and the biopsy between the EMLA group and the placebo group

\begin{tabular}{|c|c|c|c|c|c|c|c|c|}
\hline & \multicolumn{4}{|c|}{ Injection } & \multicolumn{4}{|c|}{ Biopsy } \\
\hline & \multicolumn{2}{|c|}{ Patient } & \multicolumn{2}{|c|}{ Physician } & \multicolumn{2}{|c|}{ Patient } & \multicolumn{2}{|c|}{ Physician } \\
\hline & EMLA & Placebo & EMLA & Placebo & EMLA & Placebo & EMLA & Placebo \\
\hline No pain & 8 & 3 & 12 & 4 & 24 & 21 & 23 & 26 \\
\hline Mild pain & 16 & 14 & 13 & 15 & 1 & 7 & 5 & 5 \\
\hline Moderate pain & 3 & 8 & 3 & 10 & 3 & 3 & 0 & 1 \\
\hline Severe pain & 1 & 7 & 0 & 3 & 0 & 1 & 0 & 0 \\
\hline $\begin{array}{l}\text { Exact-trend } \\
\text { test } p \text { value }\end{array}$ & \multicolumn{2}{|c|}{0.004} & \multicolumn{2}{|c|}{0.001} & \multicolumn{2}{|c|}{0.226} & \multicolumn{2}{|c|}{0.781} \\
\hline
\end{tabular}

Rotterdam. The study group consisted of children who required a skin biopsy procedure for investigation of a skin disorder. The children's parents gave signed informed consent after they had received verbal and written information about the study.

The study was randomized, double blinded, and placebo controlled. Two parallel groups were used; 31 children received an EMLA patch and 32 children received a placebo patch at the site of the biopsy for 60 minutes. After removal of the patch the physician examined the skin for side effects and then infiltrated the skin with $1 \mathrm{ml}$ of lidocaine and performed the biopsy with a $4 \mathrm{~mm}$ punch.

The child and the physician indicated the level of pain on a verbal scale as "no pain," "mild pain," "moderate pain," or "severe pain." The child also indicated the level of pain on a $10 \mathrm{~mm}$ ungraded line (visual analogue scale [VAS]). An indication of $0 \mathrm{~mm}$ represented "no pain" and $10 \mathrm{~mm}$ represented "the worst pain you can imagine." The VAS is used extensively and is considered to be as reliable as an objective pain score. It is suitable for use with subjects 4 years of age and older. ${ }^{10,11}$ The pain was scored after the lidocaine was injected and after the biopsy was performed.

The difference in the distribution of pain scores between groups was tested with use of the MannWhitney test for the VAS scores because of their posi- tive skewness. The four-point verbal pain scores were compared between groups in a $4 \times 2$ cross table and tested with use of an exact-trend test.

\section{RESULTS}

Sixty-three children were randomly recruited into the study. Three children were withdrawn from the study because two had excessive anxiety and scores were missing for one child. Thus 60 children were evaluated.

The study included 29 girls (15 in the EMLA group and 14 in the placebo group) and 31 boys (13 in the EMLA group and 18 in the placebo group). The age of the subjects varied from 4 years to 15 years (mean, 9.6 years) in the EMLA group and from 4 years to 18 years (mean, 8.5 years) in the placebo group. The groups were balanced with regard to the ages and gender of the subjects.

Tables I and II show the characteristics of the distributions of the VAS pain scores and verbal pain scores for the injection and for the biopsy in the EMLA group and the placebo group. The distribution of VAS pain scores for the injection in the EMLA group was significantly lower than in 
the placebo group $(z=3.06 ; p=0.002)$. The VAS scores for the biopsy did not show a significant difference $(z=1.47 ; p=0.142)$.

A significant difference existed between both treatment groups in the distribution of the verbal pain scores for the injection scored by the patient and by the physician. In the EMLA group the verbal pain scores were significantly lower $(p=$ 0.004 respectively $p=0.001$, two-sided, exacttrend test). In the distribution of verbal pain scores of the biopsy no significant difference existed between the EMLA and the placebo groups.

A difference in pain scoring was noted between boys and girls in the EMLA and placebo groups. In the placebo group the girls scored significantly higher on the VAS scores of the injection than did the boys $(z=2.11 ; p=0.035)$. In this group there was no difference in VAS scores of the biopsy. In the EMLA group the girls scored higher on the VAS than the boys, although the difference was not significant (injection: $z=1.69, p=0.091$; biopsy: $\mathrm{z}=1.85, p=0.065$ ).

On the verbal scales of the patient and the physician for the injection in the EMLA group, the girls scored significantly higher than the boys ( $p=0.017$ resp. $p=0.044$, two-sided, exact-trend test). The other verbal scores showed a trend of girls scoring higher than boys, but the differences were not significant.

No serious side effects were observed in any of the children.

\section{DISCUSSION}

The results of this study showed that the anesthetic patch significantly reduced the pain at the site of lidocaine infiltration before a skin biopsy procedure was performed in children.

Thune et al. ${ }^{12}$ compared use of the anesthetic cream and infiltration with prilocaine in 51 adults. The cream was an effective local anesthetic after a mean application time of 2 hours. After a shorter application time, and in the case of a large biopsy site (mean area, $10 \mathrm{~cm}^{2}$ ) in several patients, prilocaine infiltration was necessary after the cream application to obtain adequate anesthesia. ${ }^{12}$

Jones et al. ${ }^{8}$ showed that the anesthetic cream was not effective in eliminating pain on injection of the local anesthetic when it was applied for 1 hour in 60 adults. They suggested that a longer application could improve the analgesic effect, but this would limit its use for routine procedures. Gupta and Sibbald ${ }^{9}$ found that the cream provided effective anesthesia in $87 \%$ of patients after application for 110 to 180 minutes.

The anesthetic cream may be a useful local anesthetic for children undergoing kidney biopsy procedures. Ogborn ${ }^{13}$ reported that the cream was used as a substitute for dermal infiltration of local anesthetic for eight children who underwent a percutaneous kidney biopsy procedure. Five children reported no sensation of the initial skin puncture. Only one child felt "a sharp object."

In our study no difference existed in the pain scores of the EMLA and placebo groups for the biopsy. This is not surprising because infiltration with lidocaine, although painful itself, provides adequate anesthesia. All children received lidocaine infiltration after removal of the patch.

In our study girls appeared to experience more pain than boys. This finding corroborates our observations in a previous study on use of the cream as a local anesthetic in mumps/measles/rubella (MMR) vaccination in children (manuscript submitted for publication). In contrast, Taddio et al. ${ }^{14}$ reported a lower pain score in girls than in boys.

\section{REFERENCES}

1. Cooper CM, Gerrish SP, Hardwick M, Kay R. EMLA cream reduces the pain of venepuncture in children. Eur J Anaesth 1987;4:441-8.

2. Halperin DL, Koren G, Attias D, Pellegrini E, Greenberg M, Wyss M. Topical skin anesthesia for venous, subcutaneous drug reservoir and lumbar punctures in children. Pediatrics 1989;84:281-4.

3. de Waard-van der Spek FB, Oranje AP, Lillieborg S, Hop WCJ, Stolz E. Treatment of molluscum contagiosum under analgesia with a lidocaine/prilocaine cream (EMLA). J Am Acad Dermatol 1990;23:685-8.

4. de Waard-van der Spek FB, van den Berg GM, Oranje AP. EMLA cream: an improved local anesthetic-review of current literature. Pediatr Dermatol 1992;9:126-32.

5. Oranje AP, de Waard-van der Spek FB. Use of EMLA ${ }^{R}$ cream in dermatosurgical interventions of skin and genital mucosa. In: Koren G. The eutectic mixture of local anesthetics (EMLA): a breakthrough in skin anesthesia. New York: Marcel Dekker; 1995. p. 123-36.

6. Bjerring P, Arent-Nielsen L. Depth and duration of skin analgesia to needle insertion after topical application of EMLA cream. Br J Anaesth 1990;64:173-7.

7. Juhlin L, Evers H, Broberg F. A lidocaine-prilocaine cream for superficial skin surgery and painful lesions. Acta Derm Venereol (Stockh) 1980;60:544-6.

8. Jones SK, Handfield-Jones S, Kennedy CTC. Does EMLA reduce the discomfort associated with localanaesthetic infiltration? Clin Exp Dermatol 1990; 15: 177-9.

9. Gupta AK, Sibbald RG. Eutectic lidocaine/prilocaine 5\% 
cream and patch may provide satisfactory analgesia for excisional biopsy or curettage with electrosurgery of cutaneous lesions. J Am Acad Dermatol 1996;35:419-23.

10. Scott PJ, Ansell BM, Huskisson EC. Measurement of pain in juvenile chronic polyarthritis. Ann Rheum Dis 1977;36:186-7.

11. Sriwatanakul K, Kelvie W, Lasagna L, et al. Studies with different types of visual scales for measurement of pain. Clin Pharmacol Ther 1983:34:234-9.
12. Thune $P$, Faerden F, Minor BG. The analgesic effect of EMLA cream for skin biopsies. J Dermatol Treat 1990; $1: 239-41$.

13. Ogborn G. The use of a eutectic mixture of local anesthetic in pediatric renal biopsy. Pediatr Nephrol 1992;6:276- 7 .

14. Taddio A, Nulman I, Goldbach M, Ipp M, Koren G. Use of lidocaine- prilocaine cream for vaccination pain in infants. J Pediatr 1994;124:643-8

\section{CALL FOR PATIENTS WITH INHERITED DISEASES OF THE SKIN}

We in San Francisco as well as skin biologists in other cities are interested in finding the chromosomal location and eventually identifying the genes whose abnormalities underlie several inherited diseases of the skin. Initially, the group believes diseases for which this strategy offers the most likely insights include several disordors of keratinization (ichthyoses, Darier's disease, and Hailey-Hailey disease), several forms of epidermolysis bullosa, several forms of Ehlers-Danlos syndrome, psoriasis, atopic dermatitis, and keloids. Indeed, ongoing studies of several such diseases have already been fruitful.

We are eager to identify kindreds with these conditions-larger kindreds for the more common disorders (e.g., psoriasis, atopic dermatitis), and smaller as well as larger kindreds for the less common disorders (e.g., Darier's disease). If you know of such a kindred, please contact Ervin Epstein, Jr., MD, Room 269, Bldg. 100, 1001 Potrero St., San Francisco, CA 94110; telephone: 1-800-285-1267; fax: (415)282-5998.

Investigators interested in collaborating more actively in such a linkage analysis at either the clinical or the laboratory level also are most welcome to contact Dr. Epstein. 